\title{
Single cell fluorescence imaging of glycan uptake by intestinal bacteria
}

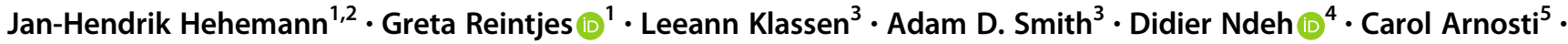 \\ Rudolf Amann ${ }^{1}$. D. Wade Abbott ${ }^{3}$
}

Received: 20 February 2018 / Revised: 3 December 2018 / Accepted: 27 February 2019 / Published online: 1 April 2019

(c) The Author(s) 2019. This article is published with open access

\begin{abstract}
Microbes in the intestines of mammals degrade dietary glycans for energy and growth. The pathways required for polysaccharide utilization are functionally diverse; moreover, they are unequally dispersed between bacterial genomes. Hence, assigning metabolic phenotypes to genotypes remains a challenge in microbiome research. Here we demonstrate that glycan uptake in gut bacteria can be visualized with fluorescent glycan conjugates (FGCs) using epifluorescence microscopy. Yeast $\alpha$-mannan and rhamnogalacturonan-II, two structurally distinct glycans from the cell walls of yeast and plants, respectively, were fluorescently labeled and fed to Bacteroides thetaiotaomicron VPI-5482. Wild-type cells rapidly consumed the FGCs and became fluorescent; whereas, strains that had deleted pathways for glycan degradation and transport were non-fluorescent. Uptake of FGCs, therefore, is direct evidence of genetic function and provides a direct method to assess specific glycan metabolism in intestinal bacteria at the single cell level.
\end{abstract}

Polysaccharides are an important food component for intestinal microbes and support genetic diversity within the

These authors contributed equally: Jan-Hendrik Hehemann, Greta Reintjes

Supplementary information The online version of this article (https:// doi.org/10.1038/s41396-019-0406-z) contains supplementary material, which is available to authorized users.

Jan-Hendrik Hehemann

jheheman@mpi-bremen.de

$\triangle$ D. Wade Abbott

wade.abbott@agr.gc.ca

1 Max Planck-Institute for Marine Microbiology, 28359

Bremen, Germany

2 Center for Marine Environmental Sciences, University of Bremen (MARUM), 28359 Bremen, Germany

3 Lethbridge Research and Development Centre, Agriculture and Agri-Food Canada, 5403-1st Avenue South, Lethbridge, AB T1J 4B1, Canada

4 Institute for Cell and Molecular Biosciences, The Medical School Newcastle University, Framlington Place, Newcastle upon Tyne NE2 4HH, UK

5 Department of Marine Sciences, University of North Carolina, Chapel Hill, NC, USA gut microbiome [1]. The chemical energy stored in structurally diverse glycans from plants and fungal cell walls provide an extensive resource landscape for microbial adaptation [2]. When presented to the intestinal microbiome of animals, these glycans selectively enrich bacteria endowed with cognate glycan degrading pathways $[2,3]$. The fermented end-products of glycan utilization by gut bacteria are short chain fatty acids, which are host absorbable secondary metabolites and represent approximately $10 \%$ of human caloric intake [4]. Therefore, conversion of indigestible glycans into compounds for host utilization is an essential symbiotic role for gut microbes that have evolved to occupy dynamic nutrient niches. Adaptation to specific dietary glycans unfolds on time scales relevant to a human life span [5]. This suggests that glycans, and functional foods in general, can be harnessed as personalized interventions to modulate the function of the gut microbiome in ways that benefit human health, such as selecting for the growth of desired taxa [6].

Underlying resource partitioning within the gut microbiome are genome encoded pathways specific for the degradation of chemically distinct glycans. These pathways are encoded in operons known as polysaccharide utilization loci (i.e. PULs), and contain genes required for sensing, depolymerizing, and transporting glycans [3, 7]. Because PULs contribute to fundamental digestive processes within 
the host intestine, improved research methods to establish metabolic abilities present within the microbiome and to explore how microbes respond to dietary interventions are urgently needed. More specifically, although biochemical characterization of PULs from cultivable isolates has made remarkable progress in defining the molecular basis of PUL function and the broad scale diversity of PULs can be assessed with metagenomics, the field is lacking methods that rapidly assign metabolic phenotypes to genotypes on the single cell level within a microbial community.

Marine bacteria have recently been shown to selectively import fluorescently labeled polysaccharides into their periplasm [8]. In these experiments substrate-based staining was combined with single cell identification by fluorescence in situ hybridization. Here we chose the gut bacterium Bacteroides thetaiotaomicron VPI-5482 (hereafter B. theta) as a model organism to ascertain whether glycans found within human diets can be fluorescently labeled and used to visualize selective glycan metabolism. B. theta consumes yeast $\alpha-$ mannan (YM) and rhamnogalacturonan-II (RGII) (Fig. 1a, b) with proteins that are encoded by specifically adapted PULs (SI Fig. 1) [9, 10]. Remarkably, despite RGII's extensive structural complexity (SI Fig. 1D) B. theta was shown to cleave all but one of the 21 distinct linkages and utilize all but four of the liberated monosaccharides for energy [10]. These complex glycans are utilized by $B$. theta through a "selfish mechanism" [9], a feeding strategy that is thought to limit the distribution of "public goods" $[3,11]$ to other members of the community. A hallmark of the selfish uptake mechanism is that complex products generated by extracellular CAZymes are selectively imported and the majority of saccharification occurs within the confines of the periplasm. This feeding strategy underpins that Bacteroidetes SusC/D-like TonBdependent transporters can accommodate large, energy-rich polysaccharides for cellular metabolism.

To determine if selfish metabolism in $B$. theta could be leveraged for imaging, YM and RGII were conjugated at free diols with 6-aminofluorescein (FLA) using a cyanogenbromide activation chemistry [12, 13]. The products of these reaction are fluorescent glycan conjugates (FGCs) FLA-YM and FLA-RGII. B. theta was cultured on unlabeled YM and RGII to metabolically activate the cells, incubated with FLA-YM or FLA-RGII, and then visualized by epifluorescence and super-resolution microscopy. Cells treated with FGCs displayed intracellular accumulation of fluorescent signal (Fig. 1c, d); whereas, cells incubated with unlabeled glycan did not display any fluorescence (SI Fig. 2). We observed that FGC uptake was time-dependent, with some $B$. theta cells showing rapid incorporation of fluorescent signal (after minutes) and peaking at 24 and $72 \mathrm{~h}$ for FLA-YM and FLA-RGII, respectively (SI Figs. 2 and 3). These observations highlight that uptake rates of FGCs can differentiate between PULs, which may reflect the structural complexity of the imported glycan; and FGCs represent potent tools to detect and study differential mechanisms of glycan uptake and metabolism by Bacteroidetes. The differences in uptake between FLA-YM and FLA-RGII were somewhat surprising as the cells were not actively dividing at this stage of the growth, and previously, xylan and laminarin displayed similar uptake kinetics in marine Bacteroidetes [8]. Therefore, this effect may result from the unique, highly branched structures of YM and RGII (Fig. 1b, d) (SI Fig. 1B, D). Alternatively, stochastic additions of FLA conjugations may result in distinct labeling density and positional chemistries, which could effect transport efficiency [12].

Although experiments were carried out with pure cultures, we observed heterogeneity in the extent of cell fluorescence for both FLA-YM and FLA-RGII (Fig. 1c, d). Some cells were highly fluorescent while others did not show any signal. This heterogeneity might indicate that uptake efficiency differs between cells within a population, which has been frequently observed for microbes [14]. In this light, FGCs appear uniquely suited to query heterogeneity of carbohydrate metabolism between individual cells. Overlays of the fluorescent signals for cellular DNA and the FGCs revealed that FGCs concentrated around the proximity of the cell forming a halo. Additional staining with Nile Red, a dye specific for the membrane lipid bilayer, demonstrated that green and red fluorescence colocalized suggesting that the glycan accumulates within the periplasm (Fig. 2a, b). Previously, accumulation of FGCs in the periplasm was reported in marine Bacteroidetes [8] and is also supported by stimulated emission depletion (STED) microscopy (Fig. 1e) and enzyme protection assays (SI Fig. 4). These patterns of FLA-YM visualization are consistent with the selfish mode of glycan metabolism previously described for YM consumption by $B$. theta (Fig. 1f, [9]).

Next, we investigated to what extent FGC uptake relies on the presence of specific PULs. Mutant B. theta strains with targeted PUL deletions for YM (Bt $\Delta$ MAN1/2/3) [9] and the major RGII PUL (Bt $\Delta$ RGII) [10] were incubated with FLA-YM and FLA-RGII, respectively (SI Fig. 2). In both cases, PUL removal ablated FGC uptake and fluorescence. This could also be verified, for YM, by FLA signal quantification, using flow cytometry (Fig. 2c), which showed that there was a significant difference in signal intensity between the mutant and WT strains. These results confirmed that glycans are modified and/or transported by proteins encoded within the PULs [7, 15]. In the Bt $\Delta$ RGII strain, there was low-level residual staining for RGII substrates (SI Fig. 2B), which suggests the intact orphan SusC/ D-like wine-RGII-specific transporter, BT1682/BT1683, can still import FLA-RGII at basal levels and that it accommodates minimally processed forms of RGII (SI Fig. 1B 

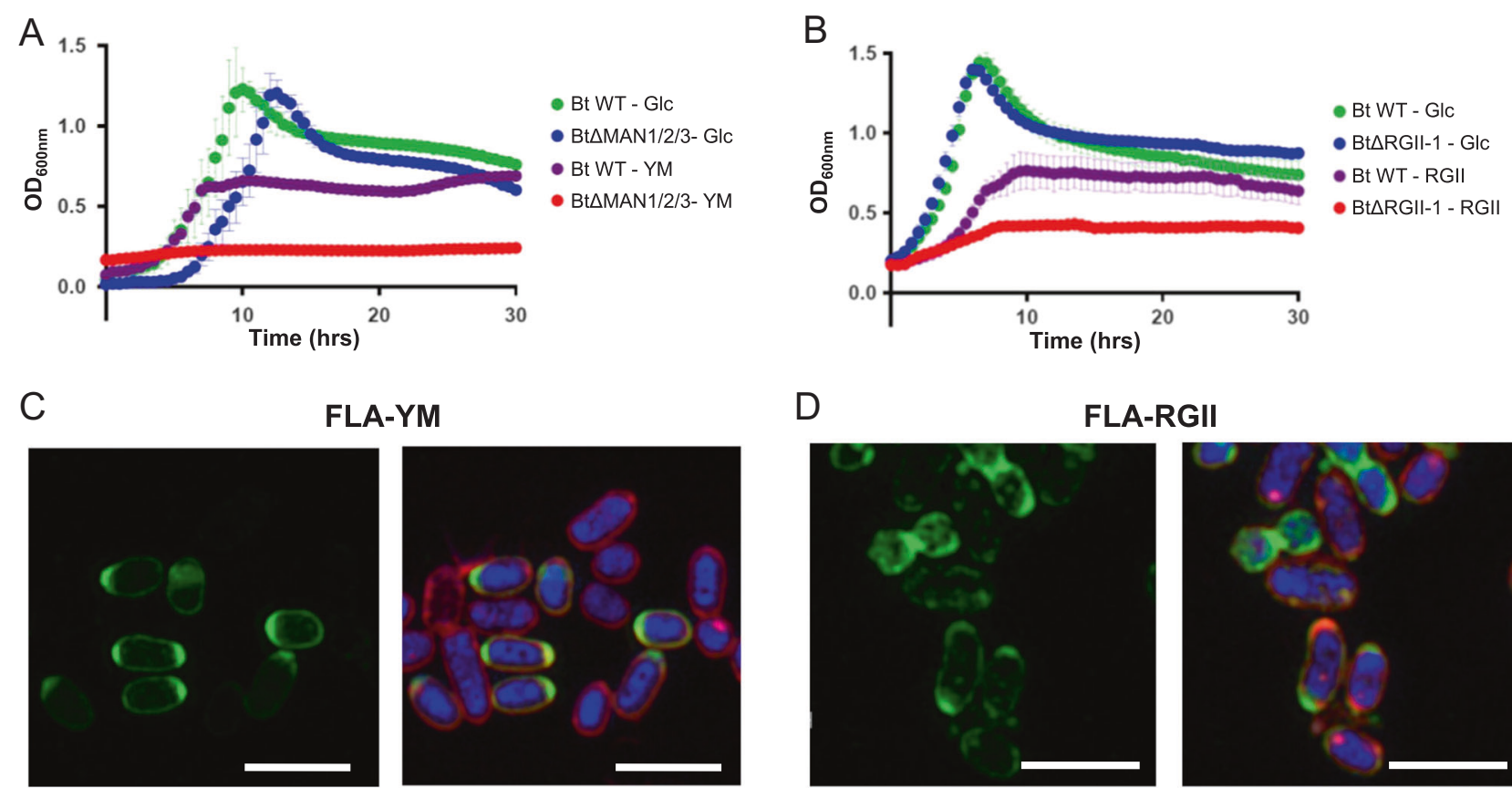

FLA-YM

D

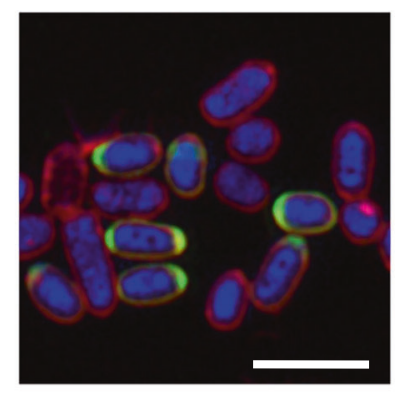

$E$
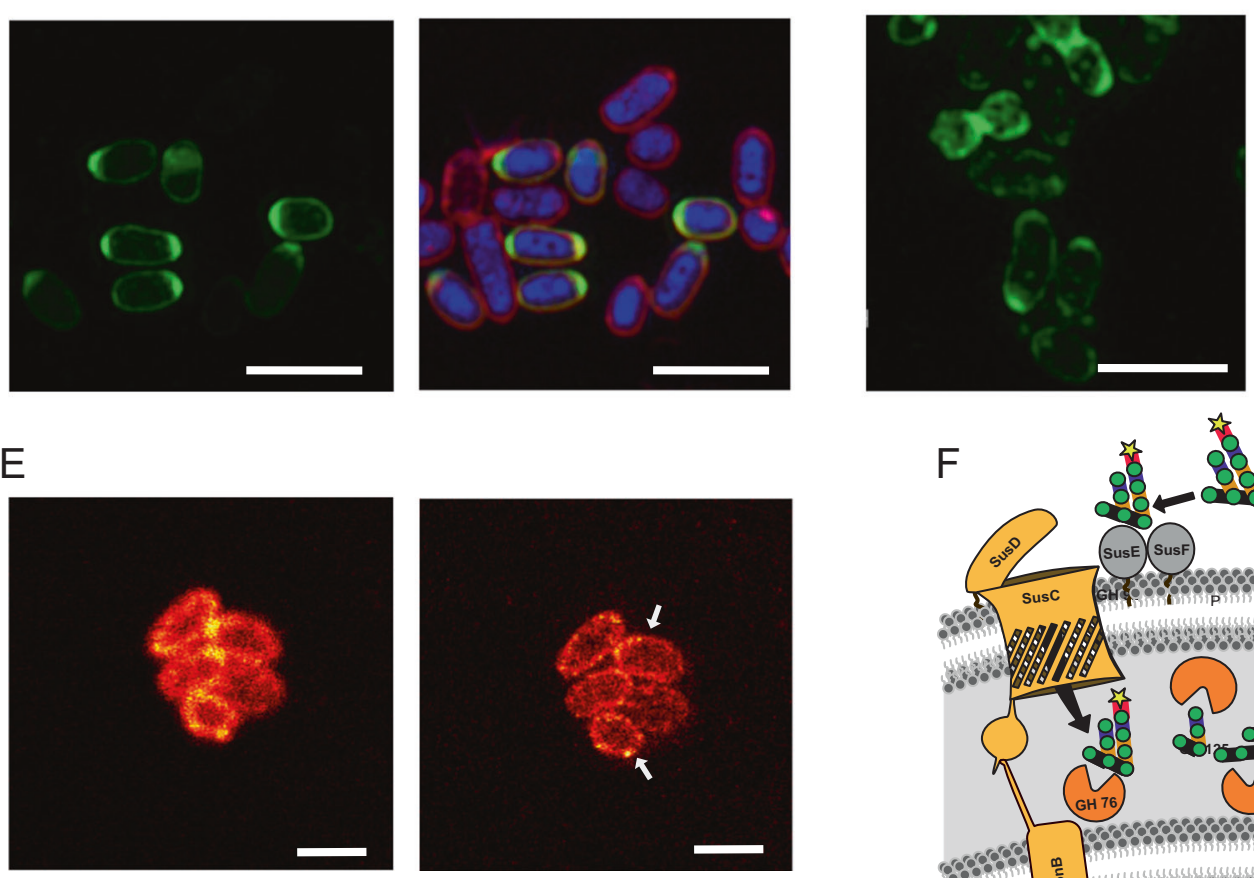

FLA-RGII

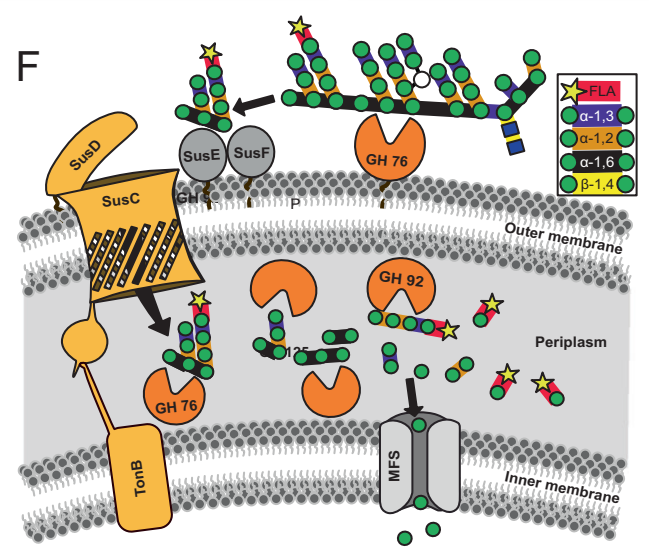

Fig. 1 Uptake of fluorescent glycans by B. theta. a Anaerobic growth profiles of $B$. theta and Bt $\Delta$ MAN1/2/3 cultured on YM and glucose. b $B$. theta and Bt $\Delta$ RGII cultured on RGII and glucose. Growth curves display the mean of five independent replicates reported every $30 \mathrm{~min}$; error bars represent s.d. c Uptake of FLA-YM and d FLA-RGII by $B$. theta. Super resolution structured illumination (SR-SIM) images of representative cells at $72 \mathrm{~h}$. Left panels are fluorescent signals from FLA-probes; right panels are overlays of DAPI, FLA-probes, and Nile Red. Scale bars represent $2 \mu \mathrm{m}$. e Uptake of FLA-YM by B. theta visualized by confocal laser scanning microscopy image (CLSM, left)

[10]). Therefore, FGCs can also be used to study preferential PUL hierarchy for engineered systems with multiple loci.

\section{Conclusion}

Glycan utilization by intestinal Bacteroidetes is critical for digestion of dietary carbohydrates. This host-symbiont interaction is facilitated by cognate PUL systems that endow bacterial strains for consumption of specific and stimulated emission depletion microscopy image (STED, right). f The illustration represents the selfish mode of yeast mannan metabolism by $B$. theta, adapted from [9]. Following limited cleavage by surface exposed GH76 enzymes, product uptake is mediated by SusC/ D-like proteins. The majority of saccharification occurs in the periplasm and is mediated by carbohydrate active enzymes spedific for the hydrolysis of $\alpha$-mannosyl linkages (e.g. GH92, GH76, and GH125). During this process FLA is transported into the periplasm, where it accumulates and can be visualized by super-resolution microscopy. Scale bar represents $1 \mu \mathrm{m}$

glycans. Despite the remarkable progress that has been made in cataloging microbiome composition from diverse sources [16] and defining the molecular basis of PUL function [2], the field still is lacking methods to rapidly assign metabolic phenotypes to genotypes in microbial communities at the single cell level. FGCs provide tools for rapid and selective study of glycanmicrobe interactions, and will assist in deciphering the mechanisms driving microbiome responses to dietary interventions [17]. 


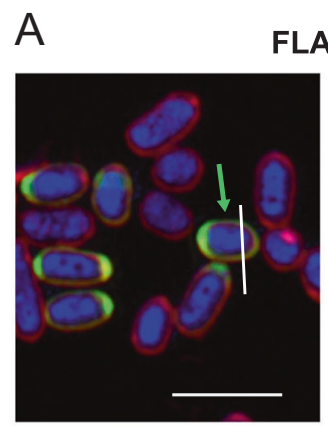

C

1)

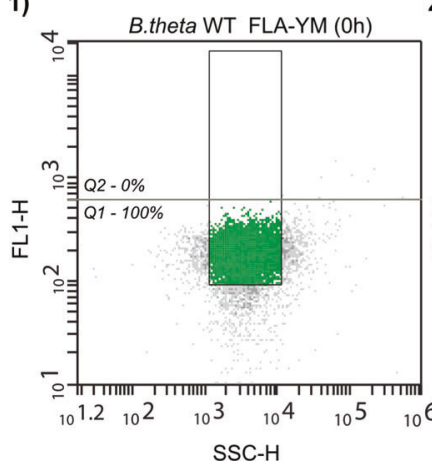

2)

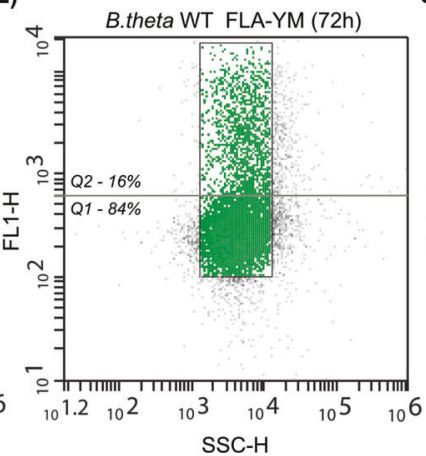

B

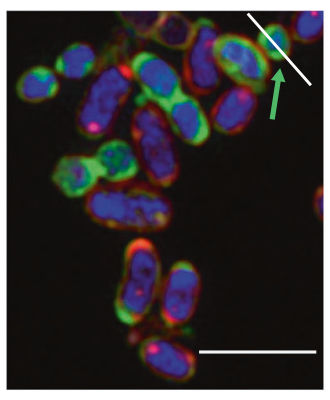

3)

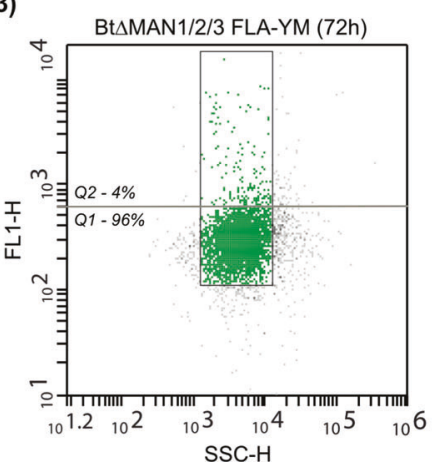

4)

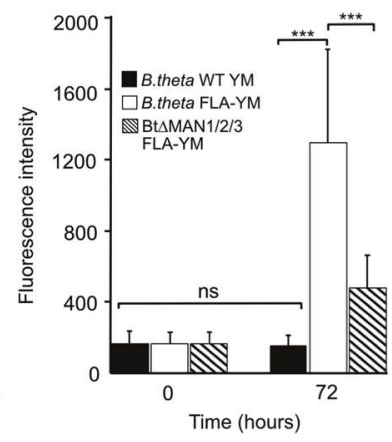

Fig. 2 Differential uptake of FGCs by individual cells in a population. a Cellular localization of DNA (DAPI), membrane (Nile Red), and FLA-YM or b FLA-RGII after $72 \mathrm{~h}$. Left panel: SR-SIM single cell images of $B$. theta cells labeled with DAPI, Nile Red, and FGC. Cells that were selected for fluorescence intensity line profiling are indicated with a green arrow and white line for profile. Right panel: Line profiles indicating fluorescence intensity at different wave lengths. Blue: DAPI; Green: FLA; and Red: Nile Red. c Quantification of FLA-YM uptake by $B$. theta and mutant $B$. theta (BtMAN1/2/3) strains over time using flow cytometry. (1) $B$. theta grown on YM shows no background fluorescence (FL1-H) signal. (2) B. theta shows significant increase in

\section{Materials and methods}

\section{Generation of FGCs}

Mannan from Saccharomyces cerevisiae (YM) prepared by alkaline extraction was purchased from Sigma (St. Louis, MO, USA; M7504). Wine RGII, purified as described previously [18]. Activation and labeling of YM and RGII was carried out as described in ref. [13], with slight modifications. Briefly, $40 \mathrm{mg}$ of polysaccharide was solubilized in $2 \mathrm{~mL}$ distilled $\mathrm{H}_{2} \mathrm{O}\left(\mathrm{dH}_{2} \mathrm{O}\right)$. To activate the polysaccharide, $30 \mathrm{mg}$ of cyanogen bromide $(\mathrm{CNBr}$;

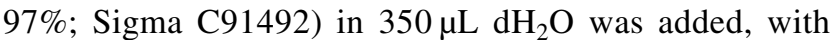
subsequent $20 \mu \mathrm{L}$ additions of $0.25 \mathrm{M} \mathrm{NaOH}$ to maintain a $\mathrm{pH}>9.5$ for $6 \mathrm{~min}$. Immediately after activation, the reaction solution was loaded onto a gel permeation chromatography column to separate excess $\mathrm{CNBr}$ from activated polysaccharide. The column $(30 \times 1 \mathrm{~cm})$, filled with Sephadex G-50 gel, was connected to a multistatic cellular fluorescence after $72 \mathrm{~h}$ incubation with FLA-YM. (3) In a $B$. theta strain with all three YM PULs deleted (Bt $\Delta$ MAN1/2/3) showed a low increase in fluorescence after $72 \mathrm{~h}$ incubation with FLA-YM. Green gating shows main cell population $(n$ (gate $)=10,000$ events). Gray line indicates percentage of cells above (Q2) and below (Q1) minimum FL1-H threshold, gated according to T0 negative sample. (4) Bar graph showing the mean fluorescence intensity of $B$. theta (white) and $\mathrm{Bt} \Delta \mathrm{MAN} 1 / 2 / 3$ (dashed) incubated with YM-FLA and a control (B. theta incubated with unlabeled YM, black) over time. $N=8500$ and error bars $=$ standard deviation. Statistical differences were calculated by Welch's $t$-test (ns: no significant difference, $* * * P<0.001$ )

peristaltic pump (Bio-Rad BioLogic LP), which set a flow rate of $1.0 \mathrm{~mL} \mathrm{~min}^{-1}$ with $0.2 \mathrm{M}$ sodium tetraborate decahydrate ( $\geq 99.5 \%, \mathrm{pH} 8.0$; Sigma S9640) as the mobile phase. Eluate was monitored by UV absorbance (280/254 nm) and recorded using Bio-Rad LP Data View software. The void volume was collected directly into vials containing $2.0 \mathrm{mg}$ fluoresceinamine isomer II (FLA; 95\%; Sigma 07985). Each vial was covered in aluminum foil to prevent decay and incubated for $24 \mathrm{~h}$ at room temperature. The labeled products were then concentrated and separated from excess FLA using Sartorius Vivaspin 15R spin columns (5000 molecular weight cut off; product no. VS15RH11). Columns were centrifuged (rcf: $210 \times g)$ at ambient temperature. Filtrate was removed and the sample was washed with $1 \mathrm{~mL}, \mathrm{dH}_{2} \mathrm{O}$. This process was repeated until the filtrate was clear of any visible orange color that is a result of FLA in solution. The purified samples were flash frozen, lyophilized, and stored at $\sim 4{ }^{\circ} \mathrm{C}$ in the dark. 


\section{FGC uptake by Bacteroides thetaiotaomicron}

Five milliliters of supplemented brain heart infusion (BHI; Difco) medium was inoculated with $B$. theta, Bt $\Delta$ MAN1/2/ 3 [9], or Bt $\Delta$ RGII [10]. Cultures were grown under anaerobic conditions $\left(85 \% \mathrm{~N}_{2}, 10 \% \mathrm{CO}_{2}\right.$, and $\left.5 \% \mathrm{H}_{2}\right)$ at $37{ }^{\circ} \mathrm{C}$ as described previously [19]. Cells were harvested at midexponential to late-exponential phase $\left(\mathrm{OD}_{600 \mathrm{~nm}} 0.6-1.0\right)$, centrifuged $(4700 \times g$ for $5 \mathrm{~min})$, and washed in $2 \times$ minimal media, which contained: $\mathrm{NH}_{4} \mathrm{SO}_{4}(8.5 \mathrm{mM}), \mathrm{Na}_{2} \mathrm{CO}_{3}(9.4$ $\mathrm{mM})$, L-cysteine-free base $(4.1 \mathrm{mM}), \mathrm{KH}_{2} \mathrm{PO}_{4} \mathrm{pH} 7.2(100$ $\mathrm{mM})$, $\mathrm{FESO}_{4} \cdot 7 \mathrm{H}_{2} \mathrm{O}(1.4 \mu \mathrm{M})$, vitamin $\mathrm{K}_{3}\left(1 \mu \mathrm{g} \mathrm{mL} \mathrm{L}^{-1}\right)$, vitamin $\mathrm{B}_{12}\left(5 \mathrm{ng} \mathrm{mL}^{-1}\right), \mathrm{NaCl}(15.4 \mathrm{mM}), \mathrm{CaCl}_{2}(0.24$ $\mathrm{mM}), \quad \mathrm{MgCl}_{2} \cdot 6 \mathrm{H}_{2} \mathrm{O} \quad(98 \mu \mathrm{M}), \quad \mathrm{MnCl}_{2} \cdot 4 \mathrm{H}_{2} \mathrm{O} \quad(50 \mu \mathrm{M})$, $\mathrm{CoCl}_{2} \cdot 6 \mathrm{H}_{2} \mathrm{O}(42 \mu \mathrm{M})$, resazurin $\left(1 \mu \mathrm{gL}^{-1}\right)$, and histidine/ hematin solution $\left(2 \mu \mathrm{LL}^{-1} \mathrm{v} / \mathrm{v} ; 1.9 \mu \mathrm{M}\right.$ hematin/200 $\mu \mathrm{M}$ L-histidine; 1000x stock solution). The washing step was repeated two more times, with the final resuspension in $2 \mathrm{~mL} 1 \times \mathrm{MM}$ with $0.5 \% \mathrm{YM}$ (WT), $0.5 \%$ RGII (WT), $0.5 \%$ glucose $+\mathrm{YM}(B$. theta and Bt $\Delta \mathrm{MAN} 1 / 2 / 3)$, or $0.5 \%$ glucose + RGII (B. theta and Bt $\Delta$ RGII) as the sole carbon source (unlabeled). Cultures were incubated overnight.

After 20-24 h of incubation, the cells were centrifuged and washed three times as explained above. The final pellet was resuspended in $2 \mathrm{~mL} 2 \times \mathrm{MM}$ and was used as the inoculum. $150 \mu \mathrm{L}$ of each inoculum was added to $1 \mathrm{~mL}$ $0.2 \%$ polysaccharide (made by mixing $500 \mu \mathrm{L} 0.4 \%$ polysaccharide with $500 \mu \mathrm{L} 2 \times \mathrm{MM})$. After the washes, the overnight cultures were inoculated into the appropriate polysaccharide substrate, as follows: $B$. theta incubated in $0.5 \%$ RGII was inoculated into both $0.2 \%$ RGII and $0.2 \%$ FLA-RGII. B. theta incubated in $0.5 \%$ YM was inoculated into both $0.2 \% \mathrm{YM}$ and $0.2 \%$ FLA-YM. The Bt $\Delta$ RGII mutant incubated in $0.5 \%$ glucose + RGII overnight was inoculated into both $0.2 \%$ RGII and $0.2 \%$ FLA-RGII. The Bt $\triangle$ MAN1/2/3 mutant incubated in $0.5 \%$ glucose + YM was inoculated into both $0.2 \% \mathrm{YM}$ and $0.2 \%$ FLA-YM. Culture tubes were wrapped in aluminum foil to prevent decay of fluorescence.

\section{Cell fixation}

Twenty microliters of each growth cultures were aliquoted into sterile $2 \mathrm{~mL}$ screw cap tubes from the cells and resuspended in $2 \mathrm{~mL} 2 \times \mathrm{MM}(0 \mathrm{~h})$. FLA-labeled and unlabeled substrates were then added to the activated cells. $40 \mu \mathrm{L}$ samples of each treatment were taken at three time points: $1 \mathrm{~min}, 24$, and $72 \mathrm{~h}$. The samples were centrifuged (rcf: $1500 \times g$ ) for $10 \mathrm{~min}$, and the supernatants removed. To fix the cells, pellets were resuspended in $1 \mathrm{~mL}$ formaldehyde (Sigma; product no. F8775), diluted to $1 \%$ with $1 \times$ phosphate-buffered saline (PBS; pH 7.4): $137 \mathrm{mM} \mathrm{NaCl}$,
$2.7 \mathrm{mM} \mathrm{KCl}, 10 \mathrm{mM} \mathrm{Na} \mathrm{HPO}_{4}, 1.8 \mathrm{mM} \mathrm{KH}_{2} \mathrm{PO}_{4}$, and incubated overnight at $4{ }^{\circ} \mathrm{C}$. The next day, cells were centrifuged (rcf: $1500 \times g$ for $10 \mathrm{~min}$ ), pellets washed in $1 \times$ PBS, and centrifuged a final time. The supernatant was removed and the pelleted cells were stored at $4{ }^{\circ} \mathrm{C}$ until further analysis.

\section{Epifluorescence microscopy}

For epifluorescence microscopy fixed $B$. theta cells were filtered onto $25 \mathrm{~mm}$ polycarbonate filters $(0.2 \mu \mathrm{m}$ pore size $)$ using a gentle vacuum ( $<200$ mbar). The cells were then counterstained with DAPI and mounted using a Citiflour (Electron Microscopy Sciences, USA) and Vector Shield (Vector Laboratories, Germany) mounting solution (4:1). The cells were visualized on a Zeiss Axioskop 2 motplus fluorescent microscope using the Axiovision software (Zeiss, Germany).

\section{Super-resolution microscopy}

For visualization, $30 \mu \mathrm{L}$ of fixed cell culture was heat fixed at $40{ }^{\circ} \mathrm{C}$ to poly-D-lysine-coated coverslips (\#1.5, thickness $0.17 \mathrm{~mm}$ ). Subsequently, additional salts from the medium are removed by washing the coverslip in MQ.

For super-resolution structured illumination microscopy (SR-SIM) the cells were then counter-stained with $4^{\prime}, 6$ diamidino-2-phenylindole (DAPI) $\left(1 \mathrm{ng} \mu \mathrm{L}^{-1} \mathrm{WS}\right)$ and Nile red $\left(2 \mathrm{ng} \mu \mathrm{L}^{-1} \mathrm{WS}\right)$ for 10 and $25 \mathrm{~min}$, respectively. After each stain, the cells are washed in MQ and, subsequently, mounted using a 4:1 Citifluor/VectaShield mounting solution. Cells were visualized on a Zeiss ELYRA PS.1 (Carl Zeiss) using 561, 488, and $405 \mathrm{~nm}$ lasers and BP 573-613, BP 502-538, and BP 420-480 + LP 750 optical filters. Zstack images were taken with a Plan-Apochromat $\times 63 / 1.4$ oil objective and processed with the software ZEN2011 (Carl Zeiss, Germany). Fluorescence intensity line profiles of individual cells were carried out using the ZEN black software.

For STED and confocal laser scanning microscopy (CLSM) cells were mounted using Prolong Diamond (Thermo Fischer, Germany). The cells were visualized on an Abberior Instrument (Aberrior Instruments $\mathrm{GmbH}$, Germany) using, for FLA-YM detection, a $485 \mathrm{~nm}$ excitation laser, $525 / 50 \mathrm{~nm}$ detector and $595 \mathrm{~nm}$ STED laser were used, with a pinhole of $70 \mu \mathrm{m}$, a pixel size of $20 \mathrm{~nm}$, dwell time $20 \mu$ s and nine line accumulation.

\section{Enzyme protection assays}

To determine if FGCs were imported into the cells, an aliquot of FLA-YM was digested by $\alpha$-mannan-specific 
enzymes from $B$. theta $[9,20]$, including three $\alpha$ mannosidases $\quad($ BT3781 $=\alpha 1,6-G H 125 ; \quad$ BT3782 $=$ BT3990 $=\alpha 1,2-$ GH92; BT3991 $=\alpha 1,3-$ GH92) and one $\alpha$ 1,6-mannanase $($ BT3782 = GH76). Digestions were carried out with $0.2 \%$ FLA-YM and $1 \mu \mathrm{M}$ of each enzyme for $1 \mathrm{~h}$ at $37^{\circ} \mathrm{C}$ in $1 \mathrm{X} \mathrm{MM}$ ( $\mathrm{pH} 7.2$ ). Digested or undigested FLAYM was incubated with $B$. theta anaerobically at $37^{\circ} \mathrm{C}$ for $24 \mathrm{~h}$. The sample with intact FLA-YM was treated with identical enzyme conditions described above. All samples were fixed in $1 \%$ formaldehyde for $1 \mathrm{~h}$ at ambient temperature, and washed in $1 \times$ PBS buffer. Samples were stored at $4{ }^{\circ} \mathrm{C}$. Cells were analyzed by epifluorescence with constant exposure times (see above).

\section{Flow cytometry and fluorescence quantification}

Cell fluorescence due to FLA-substrate uptake was quantified in all growth cultures using an Accuri C6 flow cytometer (BD Accuri Cytometers) as described previously [8]. The 8-peak and 6-peak validation bead suspensions (Spherotech, Lake Forest, IL, USA) were used as internal references. The FCM output was analyzed using FlowJo v10.4.2 (Tree Star, USA). The FCM files were imported into FlowJo, and the main population (representing single cells) was gated (green) in the FL1-H and SSC-H view. The sample statistics (counts, mean and standard deviation) and raw FL1-H results for each event in the gate $(n=10,000)$ were exported and analyzed using the R software. Statistical differences were calculated by Welch's $t$-test in R. Additionally the percentage of total events which were above (Q2) or below (Q1) the minimum FL1-H values, gated according to controls events FL1-H was calculated.

\section{Growth profiling of $B$. theta strains}

Five milliliters of BHI media was inoculated from frozen stocks of $B$. theta, Bt $\Delta \mathrm{MAN} 1 / 2 / 3$, and Bt $\Delta$ RGII. During exponential phase, cells were centrifuged (rcf: $4700 \times g$ for $5 \mathrm{~min}$ ) and washed with $2 \times \mathrm{MM}$. The cells were diluted to $\mathrm{OD}_{600 \mathrm{~nm}} \sim 0.15$. Falcon $300 \mu \mathrm{L}$ flat-bottomed 96-well microtiter plates were used for growth curves. Each well was filled with $100 \mu \mathrm{L}$ filter sterilized substrate (1\% glucose, $1 \%$ mannose, $1 \%$ YM, $1 \%$ RG-II), as well as $100 \mu \mathrm{L}$ of inoculum $(n=6)$, to get a final concentration of $0.5 \%$ substrate and $1 \times$ MM. $100 \mu \mathrm{L}$ of sterile water was added to wells with $100 \mu \mathrm{L}$ culture as negative controls. Positive controls consisted of $180 \mu \mathrm{L}$ of $\mathrm{BHI}$ and $20 \mu \mathrm{L}$ culture. Breathe-Easy gas-permeable polyurethane membranes (Sigma-Aldrich Z380059) were used to seal the multiwell plates. An Eon microplate reader (Biotek) with Gen5 software (BioTek) was used to measure and record absorbance $(600 \mathrm{~nm})$ every $30 \mathrm{~min}$ for $48 \mathrm{~h}$. Data was analyzed using GraphPad Prism software.
Acknowledgements This work was supported by funding from the Beef and Cattle Research Council awarded to DWA (Grant: FDE.13.15). J-HH, GR, and RA were supported by the Max Planck Society. J-HH acknowledges support by the Deutsche Forschungsgemeinschaft (DFG) grant HE 7217/1-1. GR acknowledges support by a Marie Skłodowska-Curie IF-Grant (RUMIC No. 840804). CA was supported by the U.S. National Science Foundation (OCE1332881 and OCE-1736772). We wish to thank Dr. Malcolm O'Neill (Complex Carbohydrate Research Centre, University of Georgia) for providing purified wine RGII and Dr. Elizabeth Lowe (Newcastle University) for the Bt $\Delta$ MAN1/2/3 mutant.

\section{Compliance with ethical standards}

Conflict of interest The authors declare that they have no conflict of interest.

Publisher's note: Springer Nature remains neutral with regard to jurisdictional claims in published maps and institutional affiliations.

Open Access This article is licensed under a Creative Commons Attribution 4.0 International License, which permits use, sharing, adaptation, distribution and reproduction in any medium or format, as long as you give appropriate credit to the original author(s) and the source, provide a link to the Creative Commons license, and indicate if changes were made. The images or other third party material in this article are included in the article's Creative Commons license, unless indicated otherwise in a credit line to the material. If material is not included in the article's Creative Commons license and your intended use is not permitted by statutory regulation or exceeds the permitted use, you will need to obtain permission directly from the copyright holder. To view a copy of this license, visit http://creativecommons. org/licenses/by/4.0/.

\section{References}

1. El Kaoutari A, Armougom F, Gordon JI, Raoult D, Henrissat B. The abundance and variety of carbohydrate-active enzymes in the human gut microbiota. Nat Rev Microbiol. 2013;11:497-504.

2. Martens EC, Kelly AG, Tauzin AS, Brumer H. The devil lies in the details: how variations in polysaccharide fine-structure impact the physiology and evolution of gut microbes. J Mol Biol. 2014;426:3851-65.

3. Grondin JM, Tamura K, Dejean G, Abbott DW, Brumer H. Polysaccharide utilization loci: fueling microbial communities. J Bacteriol. 2017;199:pii: e00860-16.

4. Bergman EN. Energy contributions of volatile fatty acids from the gastrointestinal tract in various species. Physiol Rev. 1990;70: 567-90.

5. David LA, Maurice CF, Carmody RN, Gootenberg DB, Button JE, Wolfe BE, et al. Diet rapidly and reproducibly alters the human gut microbiome. Nature . 2014;505:559-63.

6. Barratt MJ, Lebrilla C, Shapiro HY, Gordon JI. The gut microbiota, food science, and human nutrition: a timely marriage. Cell Host Microbe. 2017;22:134-41.

7. Foley MH, Cockburn DW, Koropatkin NM. The Sus operon: a model system for starch uptake by the human gut Bacteroidetes. Cell Mol Life Sci. 2016;73:2603-17.

8. Reintjes G, Arnosti C, Fuchs BM, Amann R. An alternative polysaccharide uptake mechanism of marine bacteria. ISME J. 2017;11:1640-50

9. Cuskin F, Lowe EC, Temple MJ, Zhu Y, Cameron E, Pudlo NA, et al. Human gut Bacteroidetes can utilize yeast mannan through a selfish mechanism. Nature. 2015;517:165-9. 
10. Ndeh D, Rogowski A, Cartmell A, Luis AS, Basle A, Gray J, et al. Complex pectin metabolism by gut bacteria reveals novel catalytic functions. Nature. 2017;544:65-70.

11. Rakoff-Nahoum S, Coyne MJ, Comstock LE. An ecological network of polysaccharide utilization among human intestinal symbionts. Curr Biol. 2014;24:40-9.

12. Bartling GJ, Brown HD, Forrester LJ, Koes MT, Mather AN, Stasiw RO. A study of the mechanism of cyanogen bromide activation of cellulose. Biotechnol Bioeng. 1972;14:1039-44.

13. Arnosti C. Fluorescent derivatization of polysaccharides and carbohydrate-containing biopolymers for measurement of enzyme activities in complex media. J Chromatogr B. 2003;793:181-91.

14. Ackermann M. A functional perspective on phenotypic heterogeneity in microorganisms. Nat Rev Microbiol. 2015;13:497-508.

15. Glenwright AJ, Pothula KR, Bhamidimarri SP, Chorev DS, Basle A, Firbank SJ, et al. Structural basis for nutrient acquisition by dominant members of the human gut microbiota. Nature. 2017;541:407-11.
16. Whitaker WR, Shepherd ES, Sonnenburg JL. Tunable expression tools enable single-cell strain distinction in the gut microbiome. Cell. 2017;169:538-46 e12.

17. Varki A, Cummings RD, Esko JD, Freeze HH, Stanley P, Marth JD, et al. Symbol nomenclature for glycan representation. Proteomics. 2009;9:5398-9.

18. Pellerin P, Doco T, Vidal S, Williams P, Brillouet JM, O’Neill MA. Structural characterization of red wine rhamnogalacturonan II. Carbohydr Res. 1996;290:183-97.

19. Pluvinage B, Grondin JM, Amundsen C, Klassen L, Moote PE, Xiao Y, et al. Molecular basis of an agarose metabolic pathway acquired by a human intestinal symbiont. Nat Commun. 2018; 13:1043.

20. Suits MD, Zhu Y, Taylor EJ, Walton J, Zechel DL, Gilbert HJ, et al. Structure and kinetic investigation of Streptococcus pyogenes family GH38 alpha-mannosidase. PLoS ONE. 2010;5: e9006. 